\title{
Graphical Conversion between Compliance and Modulus, Permittivity and Electric Modulus, and Impedance and Admittance
}

\author{
Masahiro Nakanishi \\ Department of Applied Physics, The Hebrew University of Jerusalem, Bergman Building, Givat Ram, Jerusalem 91904, Israel \\ Correspondence should be addressed to Masahiro Nakanishi; masahiro.nakanishi@frontier.hokudai.ac.jp
}

Received 11 September 2014; Revised 2 November 2014; Accepted 5 November 2014; Published 25 November 2014

Academic Editor: Akihide Wada

Copyright (C) 2014 Masahiro Nakanishi. This is an open access article distributed under the Creative Commons Attribution License, which permits unrestricted use, distribution, and reproduction in any medium, provided the original work is properly cited.

Spectrometries probing relaxation and retardation phenomena, such as dielectric, mechanical, and impedance spectroscopies, often require the analyses with both susceptibilities spectra $z$ and its reciprocals $1 / z$ (e.g., complex permittivity and electric modulus, mechanical compliance and mechanical modulus, and impedance and admittance). In the present paper, the geometric relation between $z$ and $1 / z$ is derived and the procedure to convert $z$ into $1 / z$ on a Cole-Cole diagram is proposed. This method helps us to relate them intuitively and yields clearer understanding on their interrelations. Moreover, it opens the new route for the geometric approach to derive many mathematical properties of spectra. The relation between peak position of $z$ spectrum and that of $1 / z$ spectrum and the shape of spectra are discussed on the basis of this method.

\section{Introduction}

In spectrometries focusing on relaxation or retardation phenomena, such as dielectric spectroscopy [1], dynamic mechanical spectroscopy [2], and impedance spectroscopy [3], not only frequency dependent susceptibilities but also their reciprocals are often subject to consideration. In dielectric spectroscopy, for example, the complex permittivity is usually employed to discuss the dynamic nature of localized charges, such as the rotational diffusion of dipoles, while its reciprocal, electric modulus, is also employed to assess the dynamics of delocalized charges, such as the migration of ions [4-6]. This is also the case for the mechanical spectroscopy. Actually, both of the mechanical compliance and the mechanical modulus which is the reciprocal of the compliance are measured and discussed depending of the phenomena of interests [7].

Generally speaking, extremely broad frequency range can be probed in these techniques: it often exceeds 10 orders of magnitude in the frequency [1-3]. Thus, obtained broad spectra often contain a variety of the dynamic processes. Some of them are compatible with the analysis in susceptibility whereas the others may be better analyzed in its reciprocal representation. In this case, one needs to switch the quantity of consideration from one to the other depending on the purpose. Therefore, the straightforward method, which enables us to change from one to the other, helps to understand the experimental data and makes their interrelations clearer.

Graphical methods can be a powerful tool to satisfy this demand. For example, the Smith chart which is indispensably used in the field of microwave engineering [810] graphically relates the complex reflection coefficient and the load impedance and so forth. Of course, since these quantities are related by mathematically rigorous formulae, one can algebraically calculate any quantities once another quantity is given. Nevertheless, the intuitive manner provides clear perspectives of the analysis. Furthermore, it can also provide another route to analyze the mathematical properties of spectra, that is, geometric method, in addition to the conventional algebraic approaches.

A Cole-Cole diagram $[11,12]$ is one of the most frequently used representations for relaxation spectra. Briefly speaking, it is a plot of a complex quantity $z$ (or its complex conjugate, 
depending on the definition) on the complex plane, where complex permittivity, compliance, and impedance are often chosen as $z$. Plotting $z$ varying the frequency yields characteristic trajectory reflecting the phenomenon. Remarkably, some elementally models, such as single exponential relaxation function in dielectric spectra and the behavior of a parallel RC circuit in impedance spectra, describe circular trajectories as frequency is varied. An analogous diagram is also employed to plot transfer functions and is called Nyquist diagram. The parameters of the transfer function can be determined and the stability of a system can be analyzed thereby with it.

In the present paper, I propose a straightforward method to convert the spectrum of arbitrary complex quantity $z$ into its reciprocal $1 / z$ based on the graphical procedure on ColeCole diagram. Thereby, interrelation between $z$ (permittivity, compliance, and impedance) spectra and $1 / z$ (moduli, admittance) can be graphically recognized. Moreover, it also opens the route to the geometric method to assess the mathematical properties of spectra.

\section{Theory}

2.1. Definition. We consider a pair of reciprocally related complex quantities, $z$ and $1 / z$. Plotting $z$ or its complex conjugate in the complex plane yields a Cole-Cole diagram. Loci of $z$ trace a characteristic trajectory on the diagram as an internal parameter is varied (typically, frequency), reflecting the phenomenon represented by $z$.

Such pairs of reciprocally related quantities can be often found in spectroscopic measurements. Complex permittivity $\varepsilon^{*}(\omega)$ and electric modulus $M_{\varepsilon}^{*}(\omega)$ are, respectively, measures of how easy and how difficult it is to deform a material electrically. The former is defined as the electric displacement $D(\omega)$ for a unit electric field $E(\omega), D(\omega)=\varepsilon^{*}(\omega) E(\omega)$, whereas the latter is defined as the electric field generated when a unit electric displacement is imposed, $E(\omega)=$ $M_{\varepsilon}^{*}(\omega) D(\omega)$. Obviously, $\varepsilon^{*}(\omega) M_{\varepsilon}^{*}(\omega)=1$, meaning that each is the reciprocal of the other $(z$ and $1 / z)$. Similarly, mechanical compliance (shear $J^{*}(\omega)$ or tensile $D^{*}(\omega)$ ) and mechanical modulus (shear $G^{*}(\omega)$ or tensile $E^{*}(\omega)$ ) are reciprocally related as $J^{*}(\omega) G^{*}(\omega)=1$ and $D^{*}(\omega) E^{*}(\omega)=$ 1. Impedance $Z^{*}(\omega)$ and admittance $Y^{*}(\omega)$ also satisfy the reciprocal relation, $Z^{*}(\omega) Y^{*}(\omega)=1$.

From the viewpoint of the similarity of the typical trajectories on a Cole-Cole diagram, it is convenient to define generalized compliance $C_{G}^{*}(\omega)$ representing permittivity, mechanical compliance, and impedance, whereas electric modulus, mechanical modulus, and admittance are represented by generalized modulus $M^{*}(\omega)$, as summarized in Table 1. The generalized modulus is the reciprocal of the generalized compliance

$$
M^{*}(\omega)=\frac{1}{C_{G}^{*}(\omega)}
$$

and vice versa. Although both $C_{G}^{*}(\omega)$ and $M^{*}(\omega)$ can be plotted onto the Cole-Cole diagram as the complex quantity $z$, only the former situation is to be described in the following discussion to avoid redundancy.
2.2. Modulus Grids Superimposed onto the Cole-Cole Diagram. When the modulus is decomposed into real and imaginary parts as $M^{*}(\omega)=M^{\prime}+i M^{\prime \prime}$, the real and imaginary parts of the corresponding compliance $\left(C_{G}^{*}(\omega)=C_{G}^{\prime}-i C_{G}^{\prime \prime}\right)$ are

$$
\begin{aligned}
& C_{G}^{\prime}=\frac{M^{\prime}}{M^{\prime 2}+M^{\prime 2}}, \\
& C_{G}^{\prime \prime}=\frac{M^{\prime \prime}}{M^{\prime 2}+M^{\prime \prime 2}} .
\end{aligned}
$$

If the compliances at various frequencies are plotted on complex plane with $C_{G}^{\prime}$ along the horizontal axis and $C_{G}^{\prime \prime}$ along the vertical axis, the well-known Cole-Cole diagram for compliance $C_{G}^{*}(\omega)$ is yielded. Now, consider how iso$M^{\prime}$ and iso- $M^{\prime \prime}$, the lines along which $M^{\prime}$ and $M^{\prime \prime}$ are constant, appear in the Cole-Cole diagram. Sets of these iso$M^{\prime}$ and iso- $M^{\prime \prime}$ lines give a coordinate grid for the modulus $M^{*}(\omega)$ superimposed onto the Cole-Cole diagram for the compliance $C_{G}^{*}(\omega)$. These $M^{\prime}$ and $M^{\prime \prime}$ grids enable us to convert compliance to modulus graphically once a trajectory on the Cole-Cole diagram is given.

To find the iso- $M^{\prime}$ line (the trajectory along which $M^{\prime}$ is constant) for given $M^{\prime}$, substitute $M^{\prime}=m^{\prime}$ and $M^{\prime \prime}=t_{1}$ in (2a) and (2b), where $m^{\prime}$ is just a constant and $t_{1}$ is a parameter to be eliminated:

$$
\begin{aligned}
& C_{G}^{\prime}=\frac{m^{\prime}}{m^{\prime 2}+t_{1}^{2}}, \\
& C_{G}^{\prime \prime}=\frac{t_{1}}{m^{\prime 2}+t_{1}^{2}} .
\end{aligned}
$$

Equation (3a) yields

$$
\left(m^{\prime 2}+t_{1}^{2}\right) C_{G}^{\prime}=m^{\prime}
$$

and dividing (3b) by (3a) yields

$$
t_{1}=\left(\frac{C_{G}^{\prime \prime}}{C_{G}^{\prime}}\right) m^{\prime} .
$$

Substituting (3c) for $\left(3 \mathrm{a}^{\prime}\right)$ to eliminate the parameter $t_{1}$, we obtain a quadratic equation in $C_{G}^{\prime}$ and $C_{G}^{\prime \prime}$. Then, completing square leads to an equation for a circle:

$$
\left(C_{G}^{\prime}-\frac{1}{2 m^{\prime}}\right)^{2}+C_{G}^{\prime \prime 2}=\left(\frac{1}{2 m^{\prime}}\right)^{2} .
$$

The radius of the circle is $1 / 2 m^{\prime}$ and the center is at $\left(C_{G}^{\prime}, C_{G}^{\prime \prime}\right)=$ $\left(1 / 2 m^{\prime}, 0\right)$. In addition, one point of this circle is fixed at the origin, irrespective of the radius. As the value of the real part of the modulus, $m^{\prime}$, is decreased, the size of the circle increases. Therefore, iso- $M^{\prime}$ lines on the Cole-Cole diagram of compliance appear as circles whose center is on the horizontal axis with one point fixed at the origin, as shown in Figure 1.

The iso- $M^{\prime \prime}$ line (the trajectories along which $M^{\prime \prime}$ is constant) for given $M^{\prime \prime}$ can be found similarly, by substituting 
TABLE 1: Summary of the definitions of compliances and moduli. $D(\omega)$ and $E(\omega)$ are electric displacement and electric field, respectively. $e_{x y}(\omega)$ and $\sigma_{x y}(\omega)$ are shear strain and shear stress. $e_{x x}(\omega)$ and $\sigma_{x x}(\omega)$ are longitudinal strain and longitudinal stress. $V(\omega)$ and $I(\omega)$ are voltage and current.

\begin{tabular}{lcc}
\hline Compliance $C_{G}^{*}(\omega)$ & Interrelation & Modulus $M^{*}(\omega)$ \\
\hline Permittivity $\varepsilon^{*}(\omega)$ & & Electric modulus $M_{\varepsilon}^{*}(\omega)$ \\
$D(\omega)=\varepsilon^{*}(\omega) E(\omega)$ & $\varepsilon^{*}(\omega) M_{\varepsilon}^{*}(\omega)=1$ & $E(\omega)=M_{\varepsilon}^{*}(\omega) D(\omega)$ \\
Shear compliance $J^{*}(\omega)$ & & Shear modulus $G^{*}(\omega)$ \\
$e_{x y}(\omega)=J^{*}(\omega) \sigma_{x y}(\omega)$ & $J^{*}(\omega) G^{*}(\omega)=1$ & $\sigma_{x y}(\omega)=G^{*}(\omega) e_{x y}(\omega)$ \\
Tensile compliance $D^{*}(\omega)$ & & Tensile modulus $E^{*}(\omega)$ \\
$e_{x x}(\omega)=D^{*}(\omega) \sigma_{x x}(\omega)$ & $D^{*}(\omega) E^{*}(\omega)=1$ & $\sigma_{x x}(\omega)=E^{*}(\omega) e_{x x}(\omega)$ \\
Impedance $Z^{*}(\omega)$ & & Admittance $Y^{*}(\omega)$ \\
$V(\omega)=Z^{*}(\omega) I(\omega)$ & $Z^{*}(\omega) Y^{*}(\omega)=1$ & $I(\omega)=Y^{*}(\omega) V(\omega)$ \\
\hline
\end{tabular}

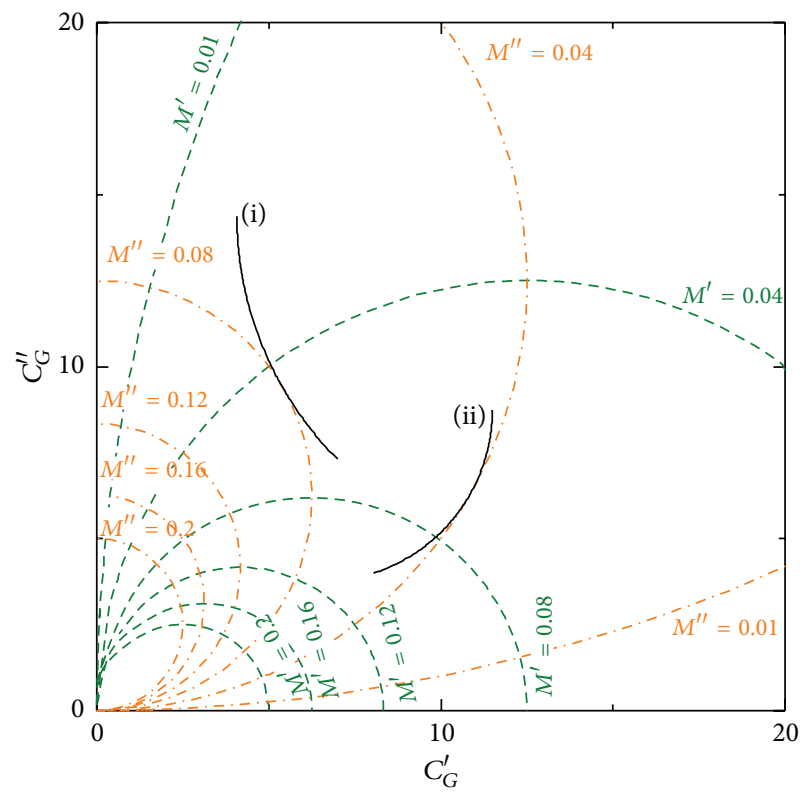

FIGURE 1: Modulus grid superimposed on a Cole-Cole diagram. The grid is composed of iso- $M^{\prime}$ and iso- $M^{\prime \prime}$ lines, along which values of $M^{\prime}$ and $M^{\prime \prime}$ are constant, respectively (see Section 2.2 for the detail). Orange dash dotted line circles indicate iso- $M^{\prime \prime}$ lines, whereas green dashed circles indicate iso- $M^{\prime}$ lines. Full line (i) indicates a trajectory circumscribed to an iso- $M^{\prime \prime}$ line, exhibiting maximum $M^{\prime \prime}$ at the tangent point. Full line (ii) indicates a trajectory inscribed to an iso$M^{\prime \prime}$ line, exhibiting minimum $M^{\prime \prime}$ at the tangent point.

$M^{\prime}=t_{2}$ and $M^{\prime \prime}=m^{\prime \prime}$ into (2a) and (2b). Iso- $M^{\prime \prime}$ lines are circles

$$
C_{G}^{\prime 2}+\left(C_{G}^{\prime \prime}-\frac{1}{2 m^{\prime \prime}}\right)^{2}=\left(\frac{1}{2 m^{\prime \prime}}\right)^{2}
$$

with radius $1 / 2 m^{\prime \prime}$ and center $\left(C_{G}^{\prime}, C_{G}^{\prime \prime}\right)=\left(0,1 / 2 m^{\prime \prime}\right)$ on the vertical axis. Again, one point of this circle lies at the origin, regardless of the radius, and the size of the circle increases as $m^{\prime \prime}$ decreases (see Figure 1).

The calculated iso- $M^{\prime}$ and iso- $M^{\prime \prime}$ lines form coordinate grids superimposed on the Cole-Cole diagram of the compliance. All these circular grid lines pass through the origin and increase in size as the value on the grid line decreases.
This modulus grid enables us to predict the appearance of the modulus spectrum for a given compliance trajectory on the Cole-Cole diagram. If the modulus is plotted on Cole-Cole diagram instead of compliance, similar circular $C_{G}^{\prime}$ and $C_{G}^{\prime \prime}$ gridlines are obtained. Therefore, the following discussion is relevant in both cases.

The Cauchy-Riemann theorem tells us that an arbitrary mapping via an analytic complex function is a conformal mapping in which the angle encompassed by an infinitesimally small triangle is preserved [13]. Conversion from modulus to compliance is also conformal mapping since the complex function employed, $f(z)=1 / z$, is analytic except at the origin. It is obvious that iso- $M^{\prime}$ and iso- $M^{\prime \prime}$ lines are orthogonal to one another on the complex plane of $M^{*}$. According to the Cauchy-Riemann theorem, this relation is conserved under mapping by the analytic function $f(z)=1 / z$, and therefore iso- $M^{\prime}$ and iso- $M^{\prime \prime}$ curves still cross orthogonally even on the complex plane of $C_{G}^{*}$. It can be seen in Figure 1 that two arbitrary intersecting circles always cross orthogonally.

\section{Examples for Applications}

With the aid of the circular modulus grids obtained above, one can obtain graphically the appearance of the modulus spectrum from the trajectory on the Cole-Cole diagram. In this section, I will present several applications for the modulus grids.

One of the major applications for the modulus grid is to find a peak in the modulus spectrum. As is clear from the circular shape of the modulus grids, maxima or minima of $M^{\prime}$ and $M^{\prime \prime}$ are at points where a circular modulus grid line is tangent to the trajectory of the compliance (lines (i) and (ii) in Figure 1). Rigorously speaking, frequency derivatives of $M^{\prime}$ or $M^{\prime \prime}$ are zero at the point where an $M^{\prime}$-grid line or an $M^{\prime \prime}$ grid line is tangent to the compliance trajectory, respectively. Moreover, if the trajectory is circumscribed to the circular grid line (line (i) in Figure 1), the frequency dependence of $M^{\prime}$ or $M^{\prime \prime}$ is concave down, whereas if the trajectory is inscribed to the circular grid (line (ii) in Figure 1), the frequency dependence of $M^{\prime}$ or $M^{\prime \prime}$ is concave up. By using these properties of the modulus grids, it is possible to find 


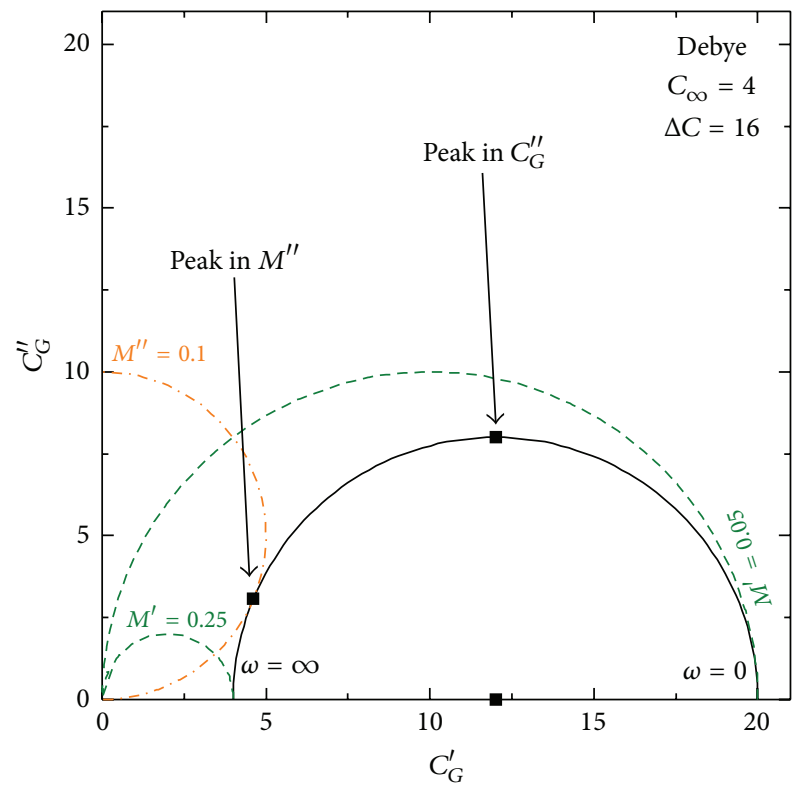

Figure 2: Cole-Cole diagram for a Debye function $\left(C_{\infty}=4\right.$ and $\Delta C=16)$ (Black full circle). An iso- $M^{\prime \prime}$ circle tangent to the trajectory of the Debye function is shown as an Orange dash dotted line circle $\left(M^{\prime \prime}=0.1\right)$. Two iso- $M^{\prime}$ circles tangent to the Debye circle are also depicted as green dashed lines $\left(M^{\prime}=0.25\right.$ and $\left.M^{\prime}=0.05\right)$. The left-hand end of the trajectory corresponds to infinite frequency whereas the right-hand end corresponds to infinitesimal frequency.

peaks of moduli graphically and even quantitatively in several special situations, as shown below.

As the simplest example, Figure 2 displays a Cole-Cole diagram of a Debye function representing the FourierLaplace transformation of a single exponential relaxation:

$$
C_{G}^{*}(\omega)=C_{\infty}+\frac{\Delta C}{1+(i \omega \tau)} .
$$

The trajectory of the Debye function describes a semicircle on the Cole-Cole diagram given by

$$
\left[C_{G}^{\prime}-\left(C_{\infty}+\frac{\Delta C}{2}\right)^{2}\right]+C_{G}^{\prime \prime 2}=\left(\frac{\Delta C}{2}\right)^{2} .
$$

Apparently, the peak in the compliance spectrum is at the top of the semicircle for this trajectory. To find the peak in the modulus spectrum, a circular $M^{\prime \prime}$-grid is drawn keeping its center along the vertical axis and a point fixed to the origin (see the orange dash dotted line in Figure 2). When the grid line is tangent to the semicircle of the trajectory of the Debye relaxation, the peak in the modulus spectrum is found at the tangent point. Then, the value of the peak height (radius of the circular $M^{\prime \prime}$-grid) can be straightforwardly calculated based on a geometrical analysis. As is clear from Figure 2, the peak in the modulus spectrum is always on the left-hand side of the semicircle of the trajectory. Taking into account that the locus on the Debye semicircle moves from right to left with increasing frequency, we can graphically confirm the well-known statement that the peak is at higher frequency in the modulus spectrum than the compliance

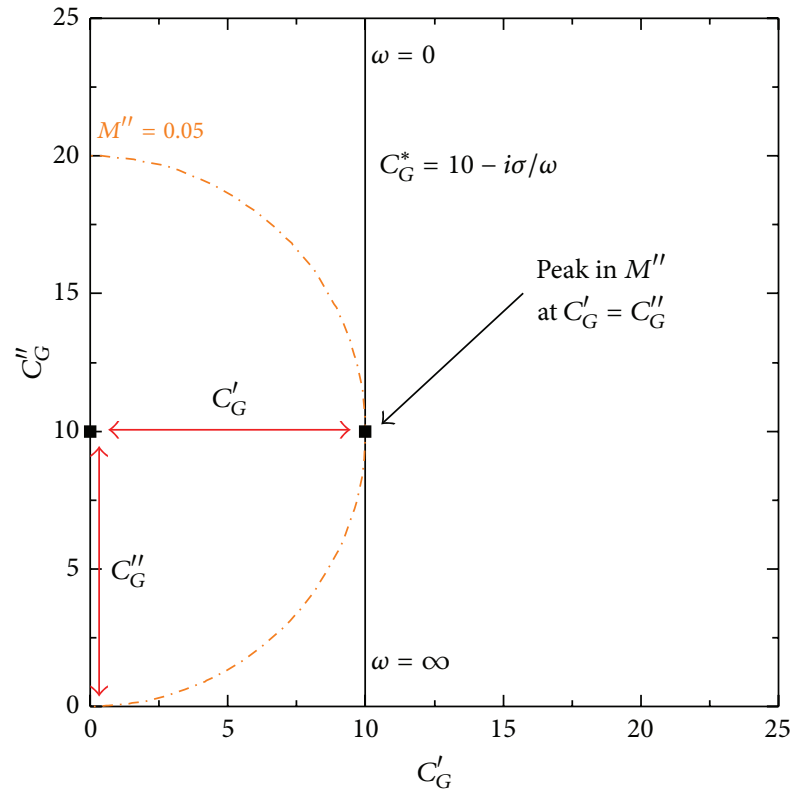

Figure 3: Cole-Cole diagram for $C_{G}^{*}(\omega)=C_{\infty}-i \sigma / \omega\left(C_{\infty}=10\right)$ (black full straight line). An iso- $M^{\prime \prime}$ circle touching the trajectory of the compliance is shown as an Orange dash dotted line circle $\left(M^{\prime \prime}=\right.$ 0.05). Since both $C_{G}^{\prime}$ and $C_{G}^{\prime \prime}$ have to coincide with the radius of the iso- $M^{\prime \prime}$ circle, the tangent point satisfies $C_{G}^{\prime}=C_{G}^{\prime \prime}$.

spectrum. Considering $M^{\prime}$-grid line which is tangent to the trajectory of the Debye relaxation, two situations are possible: one circumscribes to it and the other inscribes (see green dashed lines in Figure 2). The tangent point of the former is at infinite frequency and indicates that there is a maximum in $M^{\prime}$ at this point, while that of the latter is at zero frequency and it is seen that a minimum in $M^{\prime}$ shows up there.

As a second example, let us find an $M^{\prime \prime}$-peak for the complex compliance composed of the constants $C_{G}^{\prime}$ and the $C_{G}^{\prime \prime}$ inversely proportional to the frequency (Figure 3 ):

$$
C_{G}^{*}(\omega)=C_{\infty}-i \frac{\sigma}{\omega},
$$

where $C_{\infty}$ and $\sigma$ are parameters independent of frequency. If the generalized compliance represents complex permittivity, this represents a parallel circuit of a capacitor and resistor with constant permittivity and dc conductivity. If the compliance represents mechanical compliance, this represents a Maxwell model [2] of a spring and a dashpot connected in series. As obvious from the straight line of the trajectory drawn on the Cole-Cole diagram shown in Figure 3, the compliance spectrum exhibits neither maxima nor minima within finite frequency ranges. On the other hand, the existence of a circular $M^{\prime \prime}$-grid tangent to the straight line of the trajectory indicates that a peak shows up in the modulus spectrum. From the geometric restriction on the $M^{\prime \prime}$-grid, it is graphically proved that the peak of the modulus spectrum of (8) occurs at the frequency where $C_{G}^{\prime}=C_{G}^{\prime \prime}$.

Before proceeding with further examples, I will state a theorem that is useful for finding modulus peaks. When the trajectory on the Cole-Cole diagram has a circular shape, 


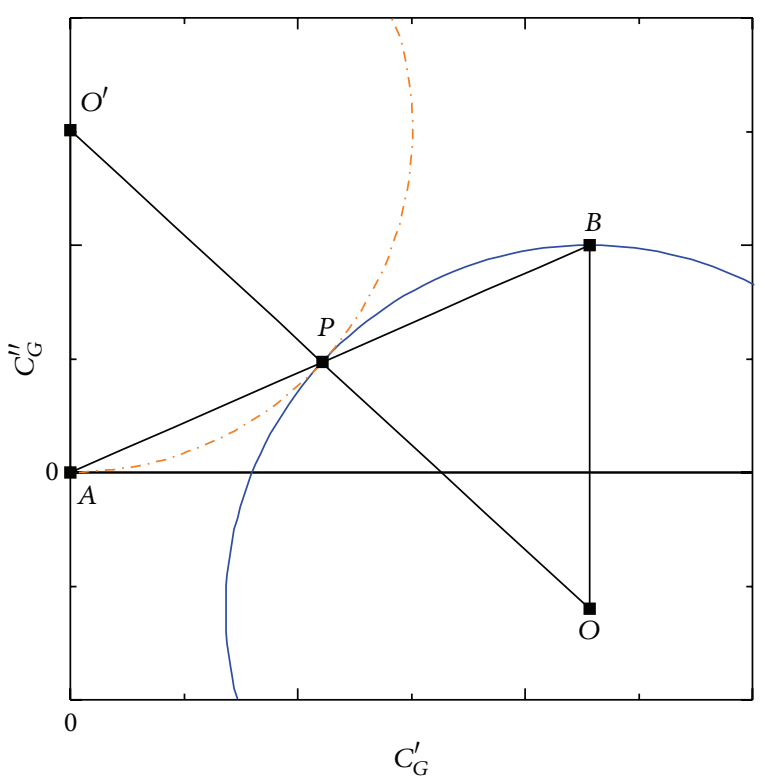

Figure 4: Proof that the straight line from the top $(B)$ of the trajectory circle with center $O$ to the origin $(A)$ passes through the tangent point $(P)$ between the iso- $M^{\prime \prime}$ circle with center $O^{\prime}$ and the trajectory circle.

the peak position of the modulus spectrum can be found via a routine procedure, irrespective of the position of the center of the circle. Such circular trajectories are found in a variety of cases: not only the Debye function but also the Cole-Cole function (CC function) [14] and Van VleckWeisskopf function (VW function) [15-17]. The statement of this theorem is as follows.

Theorem 1. When the trajectory on the Cole-Cole diagram is circular, the peak of $M^{\prime \prime}$ appears at the intersection of the circle and the straight line running from the top of the circle to the origin, irrespective of the position of the circle center.

Proof. The proof, which is based on elementary geometry, is straightforward. Consider a circle centered at arbitrary position $O$ as drawn in Figure 4, and suppose that another circle with center $O^{\prime}$ and passing through the origin is tangent to the first circle at $P$. The line segment $\overline{O^{\prime} O}$ cuts both circles at the tangent point $P$. Since $\overline{A O^{\prime}} \| \overline{B O}$, alternate-interior angles are equal, $\angle A O^{\prime} P=\angle B O P$. Therefore, the isosceles triangles $\triangle A O^{\prime} P$ and $\triangle B O P$ are homologous to one another. It follows that $\angle O^{\prime} P A=\angle O P B$. Since vertically opposite angles, $\angle O^{\prime} P A$ and $\angle O P B$, are equal, line segments $\overline{A P}$ and $\overline{P B}$ turn out to be a continuous straight line $\overline{A B}$. Therefore, the straight line running from $B$ to $A$ crosses circle with center $O$ at the common tangent point.

Two applications of this theorem are presented as follows. Although it is less important, an analogous theorem for the $M^{\prime}$-grid can also be proved. "When the trajectory on the Cole-Cole diagram is circular, a peak of $M^{\prime}$ appears at the intersection of the circle and the straight line running from

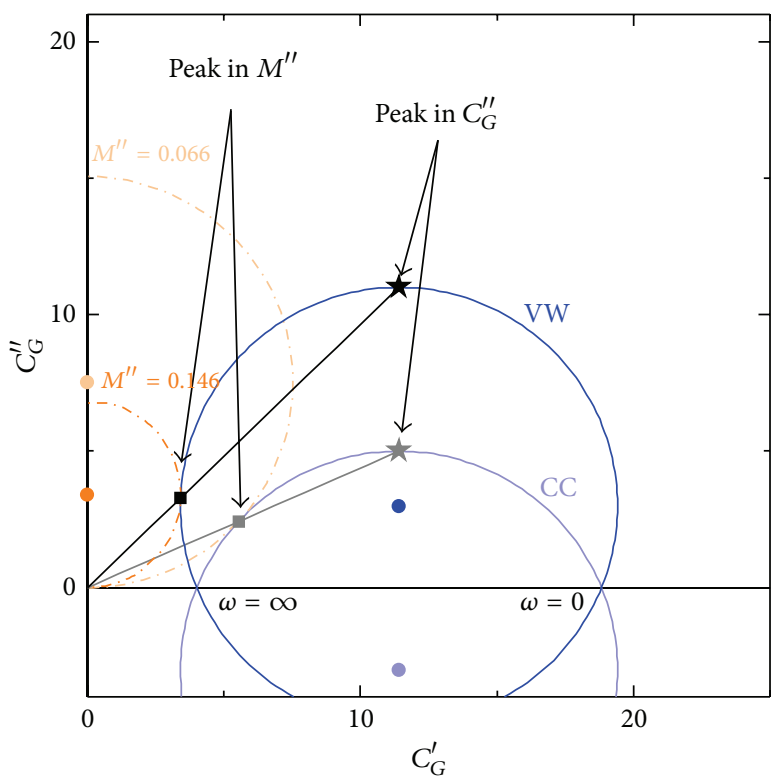

Figure 5: Cole-Cole diagram for Cole-Cole (CC) and Van VleckWeisskopf (VW) functions, which are represented as off-center circles. Dark colored lines and symbols are for the VW function, while light colored ones are for the CC function. Full curve circles stand for trajectory and dash dotted circles indicate iso- $M^{\prime \prime}$ contours. The left-hand end of a trajectory corresponds to infinite frequency whereas the right-hand end corresponds to infinitesimal frequency.

the extreme right-hand point on the circle to the origin, irrespective of the position of the circle center."

The CC function [14] is given by

$$
C_{G}^{*}(\omega)=C_{\infty}+\frac{\Delta C}{1+(i \omega \tau)^{\beta}},
$$

where $\beta$ is a parameter that controls the broadness of the spectrum. This spectrum function also has a circular trajectory on the Cole-Cole diagram although the center of the circle is in the lower half plane. The trajectory on the ColeCole diagram is

$$
\begin{aligned}
{\left[C_{G}^{\prime}\right.} & \left.-\left(C_{\infty}+\frac{\Delta C}{2}\right)\right]^{2}+\left[C_{G}^{\prime \prime}-\left(-\frac{\Delta C}{2} \cot \left(\frac{\beta \pi}{2}\right)\right)\right]^{2} \\
& =\left[\frac{\Delta C}{2} \csc \left(\frac{\beta \pi}{2}\right)\right]^{2} .
\end{aligned}
$$

Figure 5 shows an example of such a trajectory (labeled "CC"). Invoking the theorem, the peak position of the modulus spectrum can be found straightforwardly. As shown in Figure 5, by drawing a straight line from the top of the circle to the origin, the peak in modulus spectrum is found to be at the crossing point. It should be stressed that the peak was found without drawing an $M^{\prime \prime}$-grid. 
A similar analysis is also possible for the VW function which represents a vibrational mode [15-17]. The VW function is

$$
\begin{aligned}
C_{G}^{*}(\omega) & =C_{\infty}+\left(\frac{\Delta C}{2}\right)\left[\frac{1+i \omega_{0} \tau}{1+i\left(\omega+\omega_{0}\right) \tau}+\frac{1-i \omega_{0} \tau}{1+i\left(\omega-\omega_{0}\right) \tau}\right] \\
& =C_{\infty}+\int_{0}^{\infty} d t e^{-i \omega t}\left(-\frac{d}{d t}\right) \Delta C e^{-t / \tau} \cos \left(\omega_{0} t\right) .
\end{aligned}
$$

Although the trajectory of a VW function on the Cole-Cole diagram slightly deviates from a perfect circle in proximity to the horizontal axis, it is approximately circular when the vibrational frequency $\omega_{0}$ is much faster than the damping rate, $\omega_{0} \tau \gg 1$, and $\left|\ln \left(\omega / \omega_{m}\right)\right| \ll 1$,

$$
\begin{aligned}
{\left[C_{G}^{\prime}\right.} & \left.-\left(C_{\infty}+\frac{\Delta C}{2}\right)\right]^{2} \\
& +\left[C_{G}^{\prime \prime}-\left(\frac{\Delta C}{2} \cdot \frac{\left(\omega_{m} \tau\right)^{2}-1}{2\left(\omega_{m} \tau\right)^{2}-1} \omega_{m} \tau\right)\right]^{2} \\
\approx & {\left[\frac{\Delta C}{2} \cdot \frac{\left(\omega_{m} \tau\right)^{2}}{2\left(\omega_{m} \tau\right)^{2}-1} \omega_{m} \tau\right]^{2}, }
\end{aligned}
$$

where $\omega_{m}$ is the peak angular frequency in compliance, given by $\omega_{m} \tau=\sqrt{\left(\omega_{0} \tau\right)^{2}+1}$. Then the approximated circle is centered in the upper half plane on the Cole-Cole diagram representing the characteristic sigmoidal shape of the $C_{G}^{\prime}$ spectrum around the vibrational frequency. Again, the peak in the modulus spectrum can be found by drawing a straight line from top of the circular trajectory to the origin as shown in Figure 5 (labeled "VW"). Then, the peak is found at the point where the straight line crosses the circular trajectory. Furthermore, it can be clearly seen from the Cole-Cole diagram that the real part of the modulus, $M^{\prime}$, also exhibits maxima and minima that are similar to those of the $C_{G}^{\prime}$ spectrum, since it is possible to draw circular $M^{\prime}$-grid lines circumscribed and inscribed to the VW trajectory (these circles are not shown in Figure 5). This is not the case for the CC function, as $M^{\prime}$-grid lines cannot be tangent to the function's trajectory within the upper half of the Cole-Cole diagram.

The circular modulus grids tell us graphically that the peak of $M^{\prime \prime}$ always occurs at a higher frequency than that of $C_{G}^{\prime \prime}$ since circular $M^{\prime \prime}$-grid lines that are tangent to the trajectory always lie on the left-hand side of the trajectory if it has tangent point. This is one of the well-known relations between peaks in the modulus and the compliance. Additionally, the position of the tangent point relative to the center of the circular trajectory depends on the values of $C_{\infty}$ and $\Delta C$. With increasing $C_{\infty}$ (moving the circle to the right while keeping its size unchanged), the tangent point approaches the peak of $C_{G}^{\prime \prime}$, meaning that the frequency of $M^{\prime \prime}$ peak approaches that of the $C_{G}^{\prime \prime}$ peak. As $\Delta C$ is increased (making the circle bigger while keeping its left-hand end point fixed), on the other hand, the tangent point approaches the point where $\left(C_{G}^{\prime}, C_{G}^{\prime \prime}\right)=\left(C_{\infty}, C_{\infty}\right)$, resulting in infinite frequency. Furthermore, information about loss tangent can be also associated with this graphical method. On the ColeCole diagram the loss tangent, $\tan \delta=C_{G}^{\prime \prime} / C_{G}^{\prime}$, corresponds to the slope of a line from the origin to an arbitrary point on the trajectory of compliance. The maximum of the loss tangent is at the point where a straight line running from the origin is tangent to the trajectory circle. Since the straight line used to find the modulus peak ( $\overline{A B}$ in Figure 4$)$ has a lower slope than the tangent to the trajectory circle, the peak of the loss tangent lies between the peaks of the modulus and the compliance ( $P$ and $B$ in Figure 4 , resp.). Thus, we have reproduced several well-known relations between the peaks of modulus and compliance based on graphical considerations. It should be noted that if the modulus is plotted as Cole-Cole diagram instead of the compliance, typical trajectories run in the opposite direction: from left to right with increasing frequency. Then, a tangent point on the left-hand side of the trajectory means that peak of the compliance appears at a lower frequency than that of modulus peak, which is consistent with the previous results.

Although only circular trajectories have been considered above (straight line is infinitely large circle), some noncircular functions are also widely employed to reproduce experimental results: for example, Cole-Davidson (CD) function [18] and Havriliak-Negami (HN) function [19]. Reflecting their asymmetric distribution of the relaxation times, trajectories of $\mathrm{CD}$ and $\mathrm{HN}$ functions on Cole-Cole diagram draw asymmetrically skewed arcs. In these situations, the theorem to find modulus peak described above is no longer applicable because it is limited only to circular trajectories. Hence, the present graphical method enables us to make only qualitative analysis for noncircular trajectories. By drawing $M^{\prime \prime}$ grid lines, qualitative relation between peaks of compliance, modulus, and loss tangent can be straightforwardly found. More generally, one can predict the qualitative shape of the modulus from a complicated spectrum as explained in what follows. In Figure 6, superposition of two $\mathrm{HN}$ functions is plotted as Cole-Cole diagram (its frequency dependence is also shown in the inset). As is clear from the diagram, the $\mathrm{HN}$ process at higher frequency exhibits only a shoulder instead of a peak in the compliance representation. By drawing two $M^{\prime \prime}$-grid lines which tangent to the trajectory (orange dash dotted lines in Figure 6), appearance of two peaks in the modulus spectrum can be recognized. Since tangent line at lower frequency is nearly parallel to the trajectory for large range, it is estimated that the corresponding peak in the modulus is not well resolved (it is peak but close to shoulder). Such qualitative discussion is always possible irrespectively of the shape of spectra and provides clear understanding on the interrelation between processes in the modulus and those of compliance.

\section{Conclusions}

General properties of modulus grids (formed from iso- $M^{\prime}$ and iso- $M^{\prime \prime}$ lines) on the Cole-Cole diagram for compliance were studied in detail. Both iso- $M^{\prime}$ and iso- $M^{\prime \prime}$ lines are 


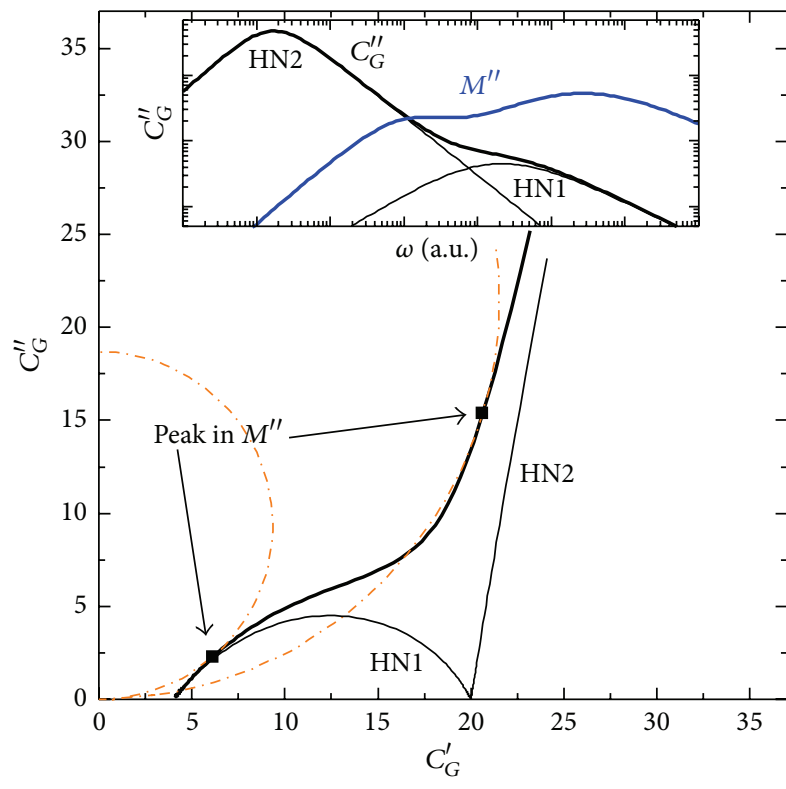

Figure 6: Cole-Cole diagram for the compliance spectrum composed of superposition of two Havriliak-Negami (HN) processes. The compliance spectrum and corresponding modulus spectrum are shown in the inset. Full bold line stands for the trajectory of the total compliance and full thin lines indicate each component (HN1 and HN2). Dash dotted circles indicate iso- $M^{\prime \prime}$ contours.

circular with one point fixed at the origin and increase in radius as the values of $M^{\prime}$ and $M^{\prime \prime}$ are decreased. This modulus grid superimposed on the Cole-Cole diagram allows us to convert graphically from compliance to modulus. Maxima and minima of $M^{\prime}$ and $M^{\prime \prime}$ correspond to the tangent points where $M^{\prime}$ and $M^{\prime \prime}$ grid lines, respectively, meet the trajectory of compliance tangentially. Therefore, the peak of the modulus can be found graphically from the trajectory. When the trajectory is a circle (Debye, Cole-Cole, or Van Vleck-Weisskopf functions), the exact position of the modulus peak can be found by drawing a straight line from top of the circle to the origin and taking the point at which the straight line intersects with the circle. With the aid of modulus grids, shapes of modulus spectra can be graphically derived once the Cole-Cole plot of the compliance has been obtained. This procedure enables us to relate compliance with modulus much easier and makes their interrelation clearer.

\section{Conflict of Interests}

The author declares that there is no conflict of interests regarding the publication of this paper.

\section{Acknowledgments}

The author thanks Dr. Alexei P. Sokolov, Dr. Yuri Feldman, and Dr. Ivan I. Popov for reading the manuscript and useful comments.

\section{References}

[1] F. Kremer and A. Schönhals, Broadband Dielectric Spectroscopy, Springer, Berlin, Germany, 2002.

[2] G. Stroble, The Physics of Polymers, Springer, Berlin, Germany, 2nd edition, 2002.

[3] E. Barsoukov and J. R. Macdonald, Impedance Spectroscopy: Theory, Experiment, and Applications, John Wiley \& Sons, Hoboken, NJ, USA, 2005.

[4] H. Wagner and R. Richert, "Dielectric relaxation of the electric field in poly(vinyl acetate): a time domain study in the range $10^{-3}-10^{6}$ s," Polymer, vol. 38, no. 2, pp. 255-261, 1997.

[5] H. Wagner and R. Richert, "Measurement and analysis of timedomain electric field relaxation: the vitreous ionic conductor 0.4 $\mathrm{Ca}\left(\mathrm{NO}_{3}\right)_{2}-0.6 \mathrm{KNO}_{3}$," Journal of Applied Physics, vol. 85, no. 3, pp. 1750-1755, 1999.

[6] M. Paluch, Z. Wojnarowska, and S. Hensel-Bielowka, "Heterogeneous dynamics of prototypical ionic glass ckn monitored by physical aging," Physical Review Letters, vol. 110, no. 1, Article ID 015702, 2013.

[7] K. Schröter and E. Donth, "Viscosity and shear response at the dynamic glass transition of glycerol," The Journal of Chemical Physics, vol. 113, no. 20, pp. 9101-9108, 2000.

[8] T. Mizuhashi, "Theory of four-terminals impedance transformation circuit and matching circuit," Journal of the Institute of Electrical Communication Engineers of Japan, vol. 177, pp. 10531058, 1937.

[9] P. H. Smith, “Transmission line calculator," Electronics, vol. 12, pp. 29-31, 1939.

[10] P. H. Smith, Electronic Applications of the Smith Chart, McGrawHill, New York, NY, USA, 1969.

[11] K. S. Cole, "Electric impedance of suspensions of spheres," Journal of General Physiology, vol. 12, pp. 29-36, 1928.

[12] K. S. Cole, "Electric phase angle of cell membranes," Journal of General Physiology, vol. 15, pp. 641-649, 1932.

[13] L. Ahlfors, Complex Analysis, McGraw-Hill, New York, NY, USA, 3rd edition, 1979.

[14] K. S. Cole and R. H. Cole, "Dispersion and absorption in dielectrics I. Alternating current characteristics," The Journal of Chemical Physics, vol. 9, no. 4, pp. 341-351, 1941.

[15] J. H. van Vleck and V. F. Weisskopf, "On the shape of collisionbroadened lines," Reviews of Modern Physics, vol. 17, no. 2-3, pp. 227-236, 1945.

[16] H. Fröhlich, Theory of Dielectrics, Oxford University Press, London, UK, 1958.

[17] R. Kubo, M. Toda, and N. Hashitsume, Statistical Physics II: Nonequilibrium Statistical Mechanics, Springer, Berlin, Germany, 2nd edition, 1991.

[18] D. W. Davidson and R. H. Cole, "Dielectric relaxation in glycerol, propylene glycol, and n-propanol," The Journal of Chemical Physics, vol. 19, no. 12, pp. 1484-1490, 1951.

[19] S. Havriliak and S. Negami, "A complex plane representation of dielectric and mechanical relaxation processes in some polymers," Polymer, vol. 8, pp. 161-210, 1967. 

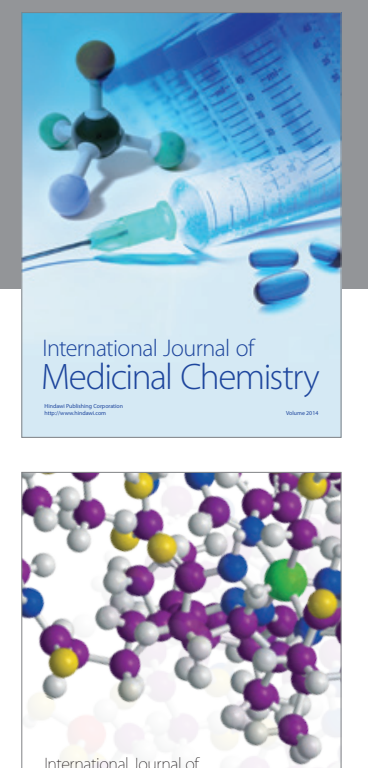

\section{Carbohydrate} Chemistry

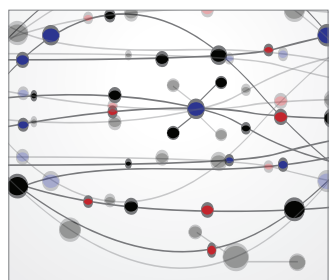

The Scientific World Journal
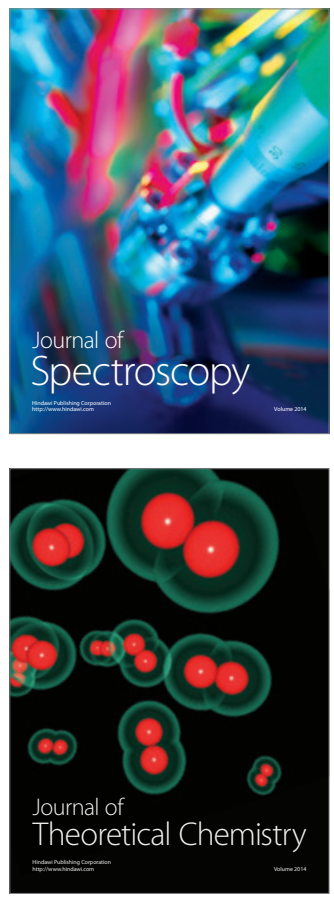
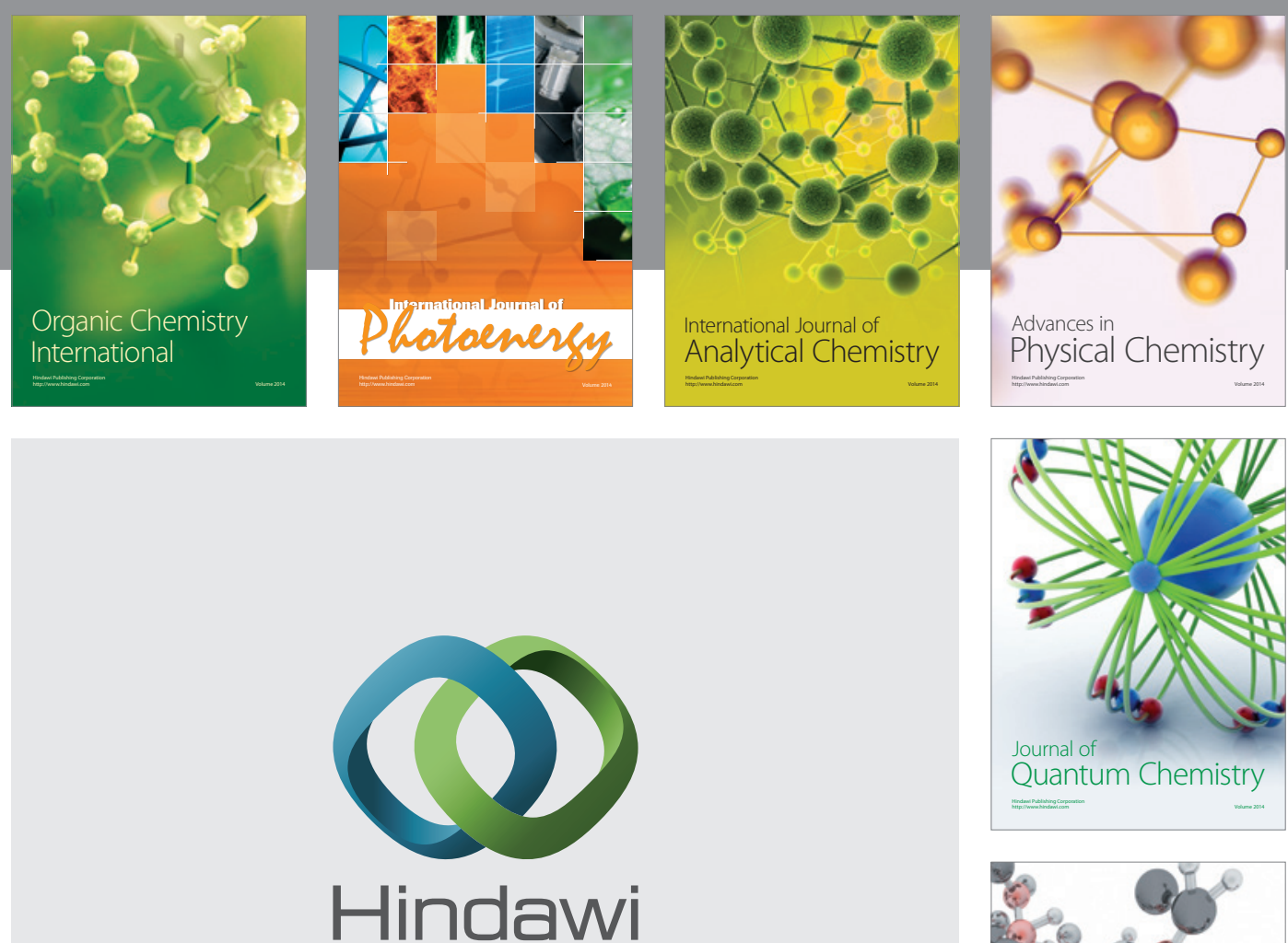

Submit your manuscripts at

http://www.hindawi.com

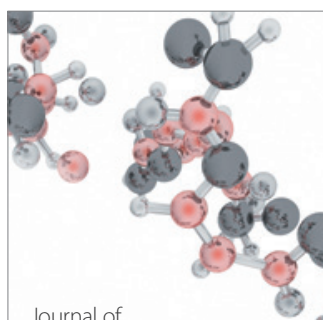

Analytical Methods

in Chemistry

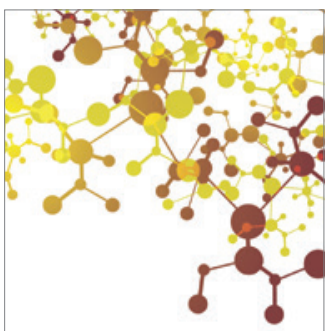

Journal of

Applied Chemistry

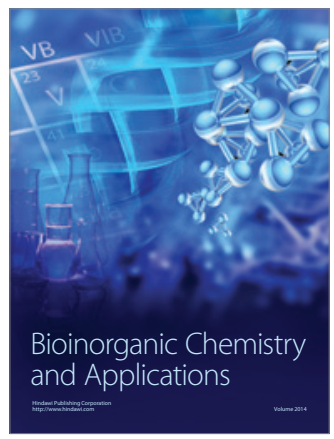

Inorganic Chemistry
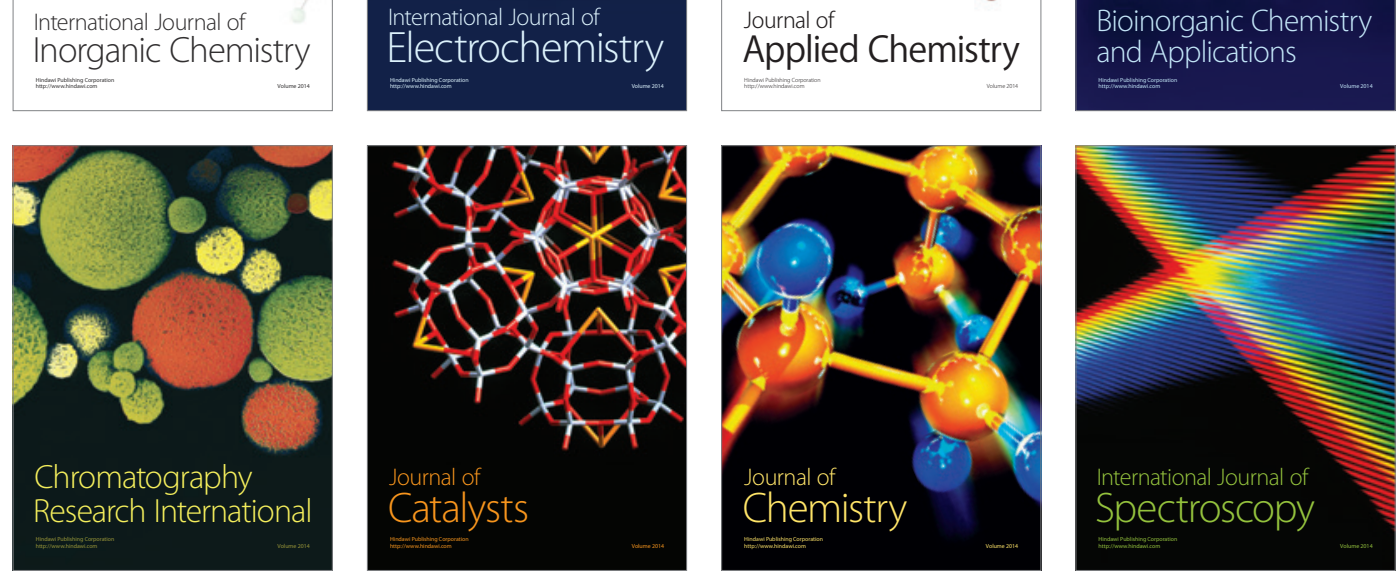